\title{
Editorial
}

\section{Quality control for the immunohistochemical demonstration of oestrogen and progesterone receptors}

It is now almost 40 years since it was first shown that oestrogenic hormones exert their actions through combination with specific receptor proteins. ${ }^{1}$ Subsequently, endocrine responsive breast cancers were shown to contain increased amounts of these oestrogen receptors (ER). ${ }^{2}$ There is almost worldwide acceptance that the measurement of oestrogen and progesterone (PR) receptors provides valuable information to aid in the selection of patients for endocrine treatment. ${ }^{3}$ Furthermore, the recent overview of randomised trials of antihormone treatments in early breast cancer has confirmed this, ${ }^{4}$ and has recognised the need for some degree of quantification, because benefit is proportional to the amount of the receptor present in the tumour. However, it has taken a switch towards the use of immunohistochemical assays in pathology laboratories ${ }^{5}$ for there to be a routine service for ER determination in all breast cancers centres within the UK.

Central to this new and important development is the establishment of appropriate external quality assessment (EQA) schemes to minimise the variability that is known to exist between laboratories when assaying ER (and PR) by immunohistochemistry on the same cases..$^{5}$ To this end, there are currently over 200 laboratories registered with the UK national external quality assessment scheme for immunocytochemistry (UK NEQAS-ICC) organising laboratory. Initial studies in this area have emphasised the need to establish appropriate assay conditions, cut off points for the immunohistochemical assays, and how the results obtained might be applied in patient management. However, little, if any, consideration has been given to how suitable the distributed paraffin wax embedded breast cancer sections are for use in EQA schemes. Thus, should we expect material derived from the organising laboratory (which collects, processes, sections, and assays breast cancer material according to its own protocol) to produce equivalent results to our own "in house" sections, which are often collected under quite different protocols and where assay optimisation is obviously biased towards the source of clinical material we normally receive? Are we thus underestimating the standards achieved by the participating laboratories and trying to set too high a standard as our EQA goal? Alternatively, is it naive to think that all breast cancer specialist centres could adopt similar or equivalent protocols for sample preparation and handling?

To evaluate these important issues, Rhodes and his colleagues on page XX of this current issue have undertaken an extensive assessment of histological sections of breast tumours that have been generated through the laboratories participating in the UK NEQAS-ICC scheme, and which show various degrees of expression of ER and $\mathrm{PR} .{ }^{6}$ Although their analysis gives us some encouragement that an acceptable degree of concordance can be reached in tumours expressing high amounts of receptor, suboptimal immunohistochemical assay sensitivity in the low to middle range of the receptor spectrum can lead to false negative results within participating laboratories. This seems to be independent of the source of EQA material, and thus could result in the selection of an inappropriate therapeutic procedure for the patient. Unfortunately, accurate receptor measurements are crucial in this area, and it is evident that problems must be overcome if we are to provide a reproducible nationwide assay service that assigns patients in a uniform manner to the correct therapeutic options.

R I NICHOLSON

Tenovus Cancer Research Centre,

Department of Pharmacology, Welsh School of Pharmacy,

University of Wales, Cardiff CF1 $3 X F$, UK

R LEAKE

Department of Biochemistry, University of Glasgow G12 8QQ, UK on behalf of the UKCCCR Biological Endpoints Group

1 Jensen EV, Jacobson HI. Basic guides to the mechanism of estrogen action. Recent Prog Horm Res 1962;18:387-414.

2 Floca PJ, Glascock RF, Irvine WT. Studies with tritium-labelled hexoestrol in advanced breast cancer: comparison of tissue accumulation of hexoestrol with response to bilateral adrenalectomy and oophorectomy. Lancet 1961; 2:796-8.

3 Nicholson RI, McClelland RA, Gee JMW. Steroid hormone receptors and their clinical significance in cancer. $\mathcal{F}$ Clin Pathol 1995;48:890-5.

4 Early Breast Cancer Trialists Collaborative Group. Tamoxifen for early breast cancer: overview of the randomised trials. Lancet 1998;351:1451-67.

5 Barnes DM, Millis RR, Beex LVA, et al. Increased use of immunohistochemistry for oestrogen receptor measurement in mammary carcinoma: the need for quality assurance. Eur $\mathcal{F}$ Cancer 1998;34:1677-82.

6 Rhodes A, Jasani B, Balaton AJ, et al. Immunohistochemical demonstration of oestrogen and progesterone receptors: correlation of standards achieved on "in house" tumours with that achieved on external quality assessment on "in house" tumours with that achieved on external quality assessment
material in over 150 laboratories from 26 countries. 9 Clin Pathol 2000;53: 292-301. 